\title{
Perfil de Solicitações ao Serviço de Informação sobre Medicamentos (SIM) de Um Hospital Materno-Infantil do Seridó Ocidental Potiguar, Brasil
}

\author{
Profile of Requests to the Drug Information Service (DIS) of a Potiguar \\ West Seridó Maternal and Child Hospital, Brazil
}

Recebido em: $13 / 01 / 2020$ Aceito em: $15 / 06 / 2020$
Adryele Gomes MAIA; Almária Mariz BATISTA Universidade Federal do Rio Grande do Norte, Escola Multicampi de Ciências Médicas do Rio Grande do Norte. Av. Dr. Carlindo Dantas, 540,

$2^{\circ}$ andar, CEP 59300-000. Caicó, RN, Brasil.

E-mail:almariamariz@yahoo.com.br

\section{ABSTRACT}

Drug Information Centers or Services play a key role in solving concrete drug use problems by providing up-to-date and reliable information. The objective of this study was to evaluate the information requests of the Drug Information Service of the Seridó Hospital in Caicó, RN, Brazil. This is an observational study conducted from March to October 2019. The study variables were the theme of the requested information, type of requestor, priority (urgent and normal), means used for request, research sources consulted for response and medicines involved. Over eight months, the Service received 28 requests, of which 53.57\% were professionals working in the service with a prevalence of $42.86 \%$ of the nursing technician. The most discussed theme was medication preparation / administration $(39.29 \%)$. The most requested means of communication was personally with $96.43 \%$, the electronic databases were the most used sources to answer requests $(47.83 \%)$ and the urgent priority represented $71.43 \%$. The analysis of the period data allowed to verify the profile of the requests fulfilled in the first months of the Service. Thus, it is remarkable that the service has been achieving its main objective of promoting the safe and rational use of medicines, reflecting its social role to the population.

Keywords: pharmaceutical care; drug information services; hospitals.

\section{RESUMO}

Os Centros/Serviços de Informação sobre Medicamentos exercem função primordial, atuando na resolução de problemas concretos ligados a uso de medicamentos e fornecendo informações confiáveis e atualizadas. O objetivo deste estudo foi avaliar as solicitações de informação do Serviço de Informações sobre Medicamentos do Hospital do Seridó em Caicó-RN. Trata-se de estudo observacional realizado no período de março a outubro de 2019. As variáveis do estudo foram temática das informações requisitadas, tipo de solicitante, prioridade (urgente e normal), meio utilizado para solicitação, fontes de pesquisa consultadas para resposta e medicamentos envolvidos. Durante oito meses o Serviço recebeu 28 solicitações, das quais 
$53,57 \%$ eram de profissionais lotados no serviço com prevalência do técnico de enfermagem com 42,86\%. O tema mais abordado foi preparo/administração de medicamentos $(39,29 \%)$. O meio de comunicação mais solicitado foi pessoalmente $(96,43 \%)$, as bases de dados eletrônicas foram as fontes mais utilizadas para responder às solicitações $(47,83 \%)$ e a prioridade urgente representou $71,43 \%$. A análise dos dados do período permitiu verificar o perfil das solicitações atendidas nos primeiros meses de funcionamento do Serviço. Assim, é notável que o Serviço vem alcançando seu objetivo principal de promover o uso seguro e racional de medicamentos, refletindo seu papel social à população.

Palavras-chave: assistência farmacêutica; serviços de informação sobre medicamentos; hospitais.

\section{INTRODUÇÃO}

Os avanços tecnológicos do último século corroboraram para descoberta e introdução de grande variedade de medicamentos no mercado. A indústria farmacêutica faz uso de diversas estratégias em prol da lucratividade, produzindo medicamentos semelhantes aos líderes de venda. Logo, o número de novos produtos cresce consideravelmente (1).

Dado o contexto, novos fármacos são disponibilizados, indicando a importância da constante atualização profissional. Perante a dificuldade de sumarizar as informações de forma mais rápida, os Centros de Informação sobre Medicamentos (CIM) foram desenvolvidos, bem como os Serviços de Informação sobre Medicamentos (SIM), visando a promoção do uso racional e seguro dos medicamentos, englobando ainda efetividade e custeio da terapêutica (2).

Cabe salientar que CIM e SIM, geralmente, são considerados sinônimos, porém ambos apresentam suas particularidades. O CIM é relacionado a estrutura física, enquanto o SIM é interligado ao tipo de atividade desempenhada. Em termos de classificação, os CIM possuem abrangência ampla, envolvendo país, região ou estado específico enquanto os SIM são restritos a instituições, como hospitais (3).

Em 1962, foi criado o primeiro CIM no departamento médico da Universidade de Kentucky, Estados Unidos. Mediante a grande aceitação dos resultados obtidos, a difusão do programa ocorreu por todo país, abrangendo também países como Canadá e diversos outros da Europa (3). O projeto pioneiro no Brasil teve registro em 1979 no Hospital Universitário Onofre Lopes, Natal, RN (4).
No ano de 1992, foi criado o Centro Brasileiro de Informação sobre Medicamentos (CEBRIM). Após dois anos, foi lançado o I Curso de Centros de Informação sobre Medicamentos, com o objetivo de capacitar profissionais farmacêuticos na implantação de CIM por todo país. O CEBRIM foi desenvolvido para servir como rede nacional dos CIM, visando a promoção de informações sobre medicamentos mediante respostas e perguntas dirigidas, informação ativa, publicações de matérias e boletins em revistas especializadas e realização de cursos e palestras, provendo informação sobre medicamentos a seus requisitantes, bem como desenvolvendo atividades estratégicas de auxílio quanto ao uso racional de medicamentos (5).

Em unidades hospitalares, CIM/SIM são de fundamental importância no uso racional de medicamentos no país, corroborando para presença do profissional farmacêutico no monitoramento das prescrições medicamentosas, fato que não é realidade em sua totalidade nas farmácias distritais, por exemplo, considerando ainda que em hospitais as equipes multidisciplinares contam com protocolos e programas informatizados que proporcionam melhorias quanto ao tipo de serviço ofertado (6).

O termo "informação sobre medicamentos", é o resultado das solicitações profissionais, bem como do público leigo, envolvendo pacientes ou usuários. As informações podem servir de base para um caso clínico de um paciente específico ou mesmo para uma população (7).

No processo de atenção à saúde, CIM/SIM exercem função primordial, atuando na resolução de problemas concretos ligados a uso de medicamentos, cedendo informações confiáveis e atualiza- 
das aos profissionais da saúde, auxiliando também no treinamento de estudantes e profissionais (8).

O Serviço de Informação sobre Medicamentos do Hospital do Seridó surgiu pela necessidade de auxiliar os profissionais de saúde do serviço e a comunidade em geral em suas dúvidas acerca de medicamentos. O SIM funciona de segunda a sexta-feira, 10 horas diárias e conta com equipe formada por 4 farmacêuticos residentes, iniciando suas atividades em março de 2019.

Dessa forma, o objetivo deste estudo foi avaliar o perfil das solicitações de informação do SIM do Hospital do Seridó.

\section{MÉTODO}

O cenário do estudo foi o Hospital do Seridó, situado no município de Caicó, RN. Configura-se como referência em Saúde Materno-Infantil para o município e cidades situadas na microrregião Seridó Potiguar. Segundo dados do Instituto Brasileiro de Geografia e Estatística (IBGE), no último censo, realizado em 2010, o município contava com 62.709 habitantes e estimou-se população de 67.554 habitantes para 2018 (9).

O hospital é de gestão municipal e atende usuários do Sistema Único de Saúde (SUS) de seu município e regiões circunvizinhas. Possui 15 leitos de obstetrícia, 12 de pediatria, 25 de clínicas médica e cirúrgica, 3 destinados a observação e 10 apartamentos, totalizando 65 leitos, o que o caracteriza como hospital de médio porte (10).

Trata-se de estudo observacional de caracterização do perfil das informações sobre medicamentos requisitadas no período de março a outubro de 2019 a partir do seu banco de dados, cujo acesso foi autorizado pelo diretor do Hospital mediante carta de anuência.

Dessa forma, as variáveis do estudo foram temática das informações requisitadas, tipo de solicitante, prioridade (urgente e normal), meio utilizado para solicitação (telefone e pessoalmente), fontes de pesquisa consultadas para resposta e medicamentos envolvidos.

A classificação farmacológica dos medicamentos envolvidos nas solicitações de informação (SI) baseou-se no sistema Anatomical Therapeutical Classification (ATC) (11). Os dados foram tabulados e analisados a partir de estatística descritiva, considerando frequências absoluta e relativa, via software Microsoft Excel.

\section{RESULTADOS E DISCUSSÃO}

Durante oito meses, o SIM recebeu 28 solicitações de informação (SI). Comparando a atividade do SIM com a de grandes Centros, o número de consultas ainda é reduzido. O CEBRIM que, pela natureza de sua atividade apresenta demanda maior de serviços, recebeu 8035 SI no período de janeiro de 2002 a dezembro de 2008 (4). Outro estudo em hospital de médio porte constatou-se, em 20 meses, 31 SI. A baixa quantidade de SI de ambos os hospitais pode ser explicada pelo tamanho reduzido dos mesmos, dispondo de poucos leitos, poucos profissionais e, assim, menor possibilidade de questionamentos, além do pouco tempo de funcionamento do SIM (12). As características das SI avaliadas estão apresentadas na Tabela 1 .

Quanto ao tipo de solicitante das SI, profissionais lotados no serviço representaram 53,57\%, destacando o técnico em enfermagem (42,86\%), seguido pelos pacientes $(25,00 \%)$, os quais foram ativos no processo de SI sobre medicamentos. Ao verificar o perfil dos solicitantes ao longo dos meses, os profissionais envolvidos no processo de prescrição e administração de medicamentos utilizaram o SIM, com ênfase para técnico em enfermagem e enfermeiro residente. Além disso, a categoria pacientes representou percentual relevante, reforçando a autonomia do sujeito e a corresponsabilidade ao longo do tratamento. Comparando-se com outros estudos foi constatado perfil de distribuição de tipos de solicitantes parecido (12-14). A acentuada diferença entre percentual de SI realizadas por enfermeiros lotados no serviço e os enfermeiros residentes pode ser interpretada pelo maior engajamento dos residentes com ações e atividades desenvolvidas pela residência multiprofissional, entre as quais, o SIM.

A maioria das SI foi recebida pessoalmente (96,43\%), o que pode ser explicado pela inserção do farmacêutico junto à equipe multiprofissional, 
com atuação clínica. Foi constatado perfil semelhante em estudo desenvolvido em hospital de Currais Novos, RN (12), representando $71 \%$ das SI pessoalmente. Independente do meio de comunicação utilizado pelo SIM para resposta às SI, estas eram registradas em formulário específico com respostas e referências e, dependendo da complexidade da resposta, os farmacêuticos iam pessoalmente ao solicitante para melhores esclarecimentos. Também foi levada em consideração a prioridade da SI, sendo $71,43 \%$ urgente e $28,57 \%$ normal. É considerada urgente quando a resposta deve ser comunicada em menos de 24 horas e normal quando a resposta pode ser comunicada em tempo superior.

O tempo para comunicação da resposta reflete diretamente na qualidade e na produtividade do atendimento. Em 2019, pesquisadores de Lisboa analisaram SI do ano de 1987 a 2017 e identificaram que o tempo para comunicação da resposta não variava muito ao longo do tempo, com cerca de $70 \%$ das SI respondidas em até 24 horas após sua recepção. No ano de 2017, 90\% das consultas foram respondidas em 24 horas (15).

Quanto à temática das SI, prevaleceu preparo/ administração de medicamentos $(39,29 \%)$, seguida de reação adversa/contraindicação $(21,43 \%)$, indicação de uso $(10,71 \%)$ e posologia/dose de medicamentos $(10,71 \%)$. O tema preparo/administração de medicamento, mais prevalente, estava relacionado com via de administração, modo de diluição e reconstituição de determinado medicamento, além de SI urgentes como itens mais prevalentes.

Tabela 1. Perfil das solicitações ao Serviço de Informações sobre Medicamentos do Hospital do Seridó em Caicó, RN (março a outubro de 2019)

\begin{tabular}{|c|c|c|}
\hline TEMA DA SOLICITAÇÃO & $\mathbf{n}$ & $\%$ \\
\hline Preparo/administração de medicamentos & 11 & 39,30 \\
\hline Reações adversas/contraindicação & 6 & 21,43 \\
\hline Indicação de uso & 3 & 10,71 \\
\hline Posologia/dose de medicamentos & 3 & 10,71 \\
\hline Conservação/estabilidade de medicamentos & 2 & 7,14 \\
\hline Interações/incompatibilidades medicamentosas & 2 & 7,14 \\
\hline Padronização & 1 & 3,57 \\
\hline Total & 28 & 100 \\
\hline TIPO DE SOLICITANTE & $n$ & $\%$ \\
\hline \multicolumn{3}{|l|}{ Profissionais lotados no serviço $(53,57 \%)$} \\
\hline Técnico de enfermagem & 12 & 42,86 \\
\hline Médico & 2 & 7,14 \\
\hline Enfermeiro & 1 & 3,57 \\
\hline Paciente $(25 \%)$ & 7 & 25,00 \\
\hline \multicolumn{3}{|l|}{ Residentes multiprofissionais $(21,43 \%)$} \\
\hline Enfermeiro & 6 & 21,43 \\
\hline Total & 28 & 100 \\
\hline PRIORIDADE & $\mathbf{n}$ & $\%$ \\
\hline Urgente & 15 & 71,43 \\
\hline Normal & 13 & 28,57 \\
\hline Total & 28 & 100 \\
\hline
\end{tabular}




\begin{tabular}{|c|c|c|c|}
\hline \multicolumn{2}{|c|}{ FONTES CONSULTADAS } & $\mathbf{n}$ & $\%$ \\
\hline \multicolumn{2}{|c|}{ Bases de dados eletrônicas } & 22 & 47,83 \\
\hline \multicolumn{2}{|c|}{ Bulário } & 14 & 30,43 \\
\hline \multicolumn{2}{|c|}{ Outros sites } & 8 & 17,40 \\
\hline \multicolumn{2}{|c|}{ Artigos científicos } & 1 & 2,17 \\
\hline \multicolumn{2}{|c|}{ Livros } & 1 & 2,17 \\
\hline \multicolumn{2}{|c|}{ Total } & 46 & 100 \\
\hline \multicolumn{2}{|r|}{$\begin{array}{l}\text { CLASSES FARMACOLÓGICAS PRESENTES NAS SOLICITAÇÕES } \\
\text { (SUBGRUPO TERAPÊUTICO) }\end{array}$} & $\mathbf{n}$ & $\%$ \\
\hline J01 & Antibacterianos de uso sistêmico & 12 & 42,86 \\
\hline $\mathrm{CO2}$ & Anti-hipertensivos & 4 & 14,29 \\
\hline N05 & Psicolépticos & 3 & 10,72 \\
\hline M01 & Anti-inflamatórios e antirreumáticos & 2 & 7,14 \\
\hline D11 & Outras preparações dermatológicas & 2 & 7,14 \\
\hline N03 & Antiepiléticos & 1 & 3,57 \\
\hline H02 & Corticosteroides de uso sistêmico & 1 & 3,57 \\
\hline V06 & Nutrientes Gerais & 1 & 3,57 \\
\hline B03 & Preparações antianêmicas & 1 & 3,57 \\
\hline- & Outros & 1 & 3,57 \\
\hline Total & & 28 & 100 \\
\hline & MEIO DE SOLICITAÇÃO & $\mathbf{n}$ & $\%$ \\
\hline Pessc & ente & 27 & 96,43 \\
\hline Telef & & 1 & 3,57 \\
\hline Total & & 28 & 100 \\
\hline
\end{tabular}

A fonte de informação mais utilizada no serviço foram as bases de dados eletrônicas (Micromedex e e-Lactancia) (47,83\%), seguida do bulário eletrônico da Agência Nacional de Vigilância Sanitária (Anvisa) (30,43\%) e outros sites (17,39\%).

Quanto à categoria outros sites, estes destinaram-se à busca de protocolos clínicos. Bases de dados como Micromedex foram oinstrumento tecnológico empregado neste estudo para identificar risco de interações medicamentosas, que podem auxiliar na fase de dispensação de medicamentos, estabelecendo uma relação direta entre uso racional de medicamentos, segurança e redução de custos. Também foi citada em outros estudos como a base de dados mais solicitada tanto no Brasil $(7,12)$ quanto na Índia (16).
Quanto aos medicamentos presentes nas SI, estes foram classificados por subgrupo terapêutico, conforme classificação ATC (11). 96,43\% dos medicamentos analisados constavam neste sistema de classificação, a exceção sendo Passiflora incarnata, classificado, então, como outros. Neste caso, predominaram antibacterianos de uso sistêmico (42,86\%), seguidos de anti-hipertensivos (14,29\%) e psicolépticos $(10,72 \%)$.

A predominância de antibacterianos de uso sistêmico pode ser explicada pela necessidade de antibioticoterapia profilática e terapêutica, sendo as principais SI sobre estabilidade, reconstituição e diluição. Em estudo realizado em hospitais de referência na Etiópia houve prevalência de antibióticos, representando $23,3 \%$ das SI (17), bem como 
em hospital da Malásia (37,8\%) (18) constatou-se perfil de uso de antimicrobianos semelhante quanto às SI em questão.

Em uma pesquisa realizada em hospital universitário pediátrico de Natal, RN a classe terapêutica mais frequente foi antimicrobianos, representando $39 \%$ das SI (19); e em estudo realizado em hospital geral de Currais Novos. RN, os pesquisadores apontaram que o subgrupo mais prevalente foi antibacterianos de uso sistêmico (23,7\%) (12), o que corrobora os resultados deste estudo. A classe anti-hipertensivos foi a segunda mais consultada, e pode ser explicado pela crescente prevalência de doenças crônicas, como hipertensão, no país (20). As SI relacionadas a psicolépticos, neste estudo, foram motivadas por necessidade de esclarecimentos quanto à segurança dos mesmos durante gestação e lactação.

Para garantir a segurança do paciente, é necessário disponibilizar aos profissionais da saúde informações técnico-científicas confiáveis e atualizadas e, neste cenário, SI constituem estratégia para atender necessidades particulares de informação, uma vez que existem diversas fontes de informa- ções sobre medicamentos em constante atualização, o que exige investimento de tempo de que nem sempre o profissional dispõe. Para tanto, o Serviço dispõe de profissionais capacitados que divulgam informação independente e pertinente às necessidades que se identifiquem $(14,21,22)$.

\section{CONCLUSÃO}

A análise dos dados permitiu verificar o perfil das SI atendidas nos primeiros meses de funcionamento do SIM, que tem potencial para atender requisitos de produtividade, resolutividade e qualidade das informações prestadas, através da análise de variáveis tais como as fontes de informação utilizadas e o fato do SIM responder a todas as SI recebidas. Além disso, foram constatadas diferenças e semelhanças entre as práticas de outros Centros/Serviços que desenvolvem atividades comuns. Assim, o Serviço vem se consolidando em seu objetivo principal de promover o uso seguro e racional de medicamentos, refletindo seu papel social à população.

\section{REFERÊNCIAS}

1. Gava CM, Bermudez JAZ, Pepe VLE, Reis ALA. Novos medicamentos registrados no Brasil: podem ser considerados como avanço terapêutico? Cien Saude Colet 2010;15(3):3403-3412. DOI: $0.1590 / \mathrm{S} 1413-$ 81232010000900015 .

2. Rapkiewicz JC, Trebien HA, Pereira JG, Lacerda RB, Paula CS. Centro de Informação sobre Medicamentos do Conselho Regional de Farmácia do Paraná: avaliação do serviço e satisfação do usuário. Rev Bras Farm. 2010;91(3):111-118.

3. Vidotti CCF, Silva EV, Hoefler R. Implantação e desenvolvimento de centro de informação sobre medicamentos em hospital como estratégia para melhorar a farmacoterapia. Pharm. Bras. 2010:1-23.

4. Santos L, Jacoby T, Zuckermann J, Martinbiancho JK, Mahmud SDP, Negretto GW, et al. Centro de informações sobre medicamentos: avaliação das informações passivas em hospital universitário no sul do Brasil. Rev. HCPA. 2009;29(3):212-217.

5. Silva EV, Castro LLC, Bevilaqua LDP, Vidotti CCF, Hoefler R. Centro Brasileño de Información de Medica- mentos (CEBRIM): caracterización del servicio y estudio de opinión de los usuarios. Rev OFIL. 2003;13(2):55-56.

6. Pasqualotto A, Bitencourt PER, Nietiedt NA, Paula LF, Nogueira RO, Gomez R. Interações entre medicamentos sujeitos a controle especial dispensados na Farmácia Distrital Centro de Porto Alegre, RS, Brasil. Rev. Infarma. 2018;30(3):146-151. DOI: 10.14450/2318-9312.v30. e3.a2018.pp146-15.

7. Nicoletti MA, Marques GRS, Aguiar PM, Storpirtis S. Diagnóstico situacional da atuação dos centros de informações sobre medicamentos no Brasil. Rev. Eletr. Farm.2017;14(1):5-14.

8. Santos L, Martinbiancho J, Tadiotto AL, Kreutz LM. Perfil das interações medicamentosas solicitadas ao centro de informações sobre medicamentos de hospital universitário. Rev HCPA. 2011;31(3):326-335. DOI: 10.5216/ ref.v14i1.42695.

9. IBGE. Instituto Brasileiro de Geografia e Estatística. Censo Demográfico do Brasil. https://cidades.ibge.gov. $\mathrm{br} / \mathrm{brasil} / \mathrm{rn} / \mathrm{caico} /$ panorama [acessado em 15 de novembro de 2019]. 
10. Maia Neto JF (Org.). Farmácia hospitalar e suas interfaces com a saúde. São Paulo: RX, 2005. 316p.

11. WHO. World Health Organization. Anatomical Therapeutic Chemical (ATC): structure and principles. Geneva: WHO; 2013. [acessado em 3 de novembro de 2019]. Disponível em: http://www.whocc.no/atc/structure_and principles/.

12. Martins ABN, Batista AM. Estruturação de um Serviço de Informações sobre Medicamentos (SIM) em um hospital do Rio Grande do Norte, Brasil. Infarma Cien Farm. 2019;31(2)121-128. DOI: 10.14450/2318-9312. v31.e2.a2019.pp121-128.

13. Silva BMS, Sá TR, Silva DPD, Pio IDSL, Dantas ACS, Nunes DM. Perfil das solicitações do centro de informação sobre medicamentos da Universidade Federal do Vale do São Francisco. Rev. Extramuros. 2018;6(1): 103-107.

14. Silva CDC, Coelho HLL, Arrais PSD, Cabral FR. Centro de informação sobre medicamentos: contribuição para o uso racional de fármacos. Cad Saúde Pública. 1997;13(3):531-535.

15. Simón A, Mendes AP. Os Centros de Informação de Medicamentos: Evolução e Perspetivas Futuras a Partir da Experiência de um Centro Nacional. Rev Port Farmacoter. 2018;10:171-180. DOI: 10.25756/rpf.v10i4.20.

16. Peter AV, Murali A, Tomy T, Londhe SP. Assessment and utilization of drug information services and creating awareness for enhanced utilization of drug information centre in a tertiary care teaching hospital. Asian J Pharm Clin Res. 2017;10(5):270-274. DOI: 10.22159/ajpcr. 2017.v10i5.17180.
17. Ashenef A, Reshid E, Yilma Z, Melaku T, Chane T. Assessment of the use and status of new drug information centers in a developing country, Ethiopia: the case of public university hospital drug information centers. BioMed Res Int. 2018:1-11. DOI: 10.1155/2018/ 3840976.

18. Ali AA, Yusoff SM, Joffry SM, Wahab MSA. Drug information service awareness program and its impact on characteristics of inquiries at DIS Unit in Malaysian public hospital. Arch Pharm Pract. 2013;4(1):9-14. DOI: 10.4103/2045-080X.111576.

19. Marinho RNA, Fernandes VMAL, Medeiros WRDB, Brasil RRF, Barroso EMA, Araújo PTB, Cabral CHK, Batista AM. Serviço de Informação Sobre Medicamentos em um Hospital Universitário Pediátrico: Análise de Dados. In: 9. Congresso Brasileiro de Farmácia Hospitalar / 2. Congresso Brasileiro de Farmácia Hospitalar. 2013.

20. Malta DC, Gonçalves RPF, Machado ÍE, Freitas MIdF, Azeredo C, Szwarcwald CL. Prevalência da hipertensão arterial segundo diferentes critérios diagnósticos, Pesquisa Nacional de Saúde. Rev Bras Epidemiol. 2018; 21(Suppl 1):1-15. DOI: 10.1590/1980-549720180021.

21. Rosenberg JM, Koumis T, Nathan JP et al. Current status of pharmacistoperated drug information centers in the United States. Am J Health-Syst Pharm. 2004; 61:20232032. DOI: 10.1093/ajhp/61.19.2023.

22. Schjøtt J, Pomp E, Gedde-Dahl A. Quality and impact of problemoriented drug information: a method to change clinical practice among physicians? Eur J Clin Pharmacol. 2002;57:897-902. DOI: 10.1007/s00228001-0386-5. 